\title{
Article
}

\section{Evaluating the Chinese Revised Controlling Behaviors Scale (C-CBS-R)}

Tiwari Elsie Ch, Agnes, Yee Tak Fong, Daniel, Ko Ling Chan, Edward, Hoi Ming Tang, Debbie and Graham-Kevan, Nicola

Available at http://clok.uclan.ac.uk/13397/

Tiwari Elsie Ch, Agnes, Yee Tak Fong, Daniel, Ko Ling Chan, Edward, Hoi Ming Tang, Debbie and Graham-Kevan, Nicola ORCID: 0000-0003-0621-3093 (2015) Evaluating the Chinese Revised Controlling Behaviors Scale (C-CBS-R). Journal of Interpersonal Violence, 30 (2). pp. 314-332. ISSN 0886-2605

It is advisable to refer to the publisher's version if you intend to cite from the work. http://dx.doi.org/10.1177/0886260514534778

For more information about UCLan's research in this area go to http://www.uclan.ac.uk/researchgroups/ and search for <name of research Group>.

For information about Research generally at UCLan please go to http://www.uclan.ac.uk/research/

All outputs in CLoK are protected by Intellectual Property Rights law, including Copyright law. Copyright, IPR and Moral Rights for the works on this site are retained by the individual authors and/or other copyright owners. Terms and conditions for use of this material are defined in the policies page. 
Evaluating the Chinese Revised Controlling Behaviors Scale (C-CBS-R)

Agnes Tiwari, Daniel Yee Tak Fong,

Edward Ko Ling Chan, Elsie Chau Wai Yan, and Gloria Ling Lee Lam

The University of Hong Kong

Debbie Hoi Ming Tang

Po Leung Kuk

Nicola Graham-Kevan

University of Central Lancashire

Mid Sweden University 


\section{Abstract}

The present study evaluated the utility of the Chinese version of the Revised

Controlling Behaviors Scale (C-CBS-R) as a measure of controlling behaviors in

violent Chinese intimate relationships. Using a mixed-methods approach, in-depth,

individual interviews were conducted with 200 Chinese women survivors to elicit

qualitative data about their personal experiences of control in intimate relationships.

The use of controlling behaviors was also assessed using the C-CBS-R. Interview

accounts suggested that the experiences of 91 of the women were consistent with the

description of coercive control according to Dutton and Goodman's (2005)

conceptualization of coercion. Using the split-half validation procedure, a receiver

operating characteristics (ROC) curve analysis was conducted with the first half of the

sample. The area under the curve (AUC) for using the C-CBS-R to identify high

control was .99 , and the cutoff score of 1.145 maximized both sensitivity and

specificity. Applying the cutoff score to the second half gave a sensitivity of $96 \%$ and

a specificity of $95 \%$. Overall, the C-CBS-R has demonstrated utility as a measure of

controlling behaviors with a cutoff score for distinguishing high from low levels of

control in violent Chinese intimate relationships.

Keywords: intimate partner violence, controlling behavior assessment, Chinese 
It has long been recognized that just counting the number of violent acts cannot adequately explain the violence in intimate relationships (for example, Dutton \& Goodman, 2005; Edleson \& Tolman, 1992). Rather, attention should be given to the context in which intimate partner violence (IPV) takes place. Specifically, it is important to ascertain whether the violence is part of a general pattern of power and control in such relationships (Johnson, 2008). However, assessing control in violent intimate relationships has been a challenge for practitioners and researchers. In particular, differentiating high from low levels of control in the relationship is problematic. Cluster analysis was used to identify clusters of high and low control individuals in previous studies (Graham-Kevan \& Archer, 2003; Johnson, 2008; Johnson \& Leone, 2005). There are, however, problems with using a cluster analysis method, including the lack of well-established rules for defining a cluster (Pinj \& Stewart, 1983) or definitive criteria for admission to a cluster (Cattell, 1978). Furthermore, the nature of the sample significantly influences the nature of the cluster (Johnson, 2008). Thus, even when the sample contains very few or no individuals with a high level of control, a cluster analysis will still find clusters of higher and lower levels of control. In such cases, a high level of control is only so in comparison with the rest of the sample. It has been suggested that both qualitative and quantitative data should be used concurrently to find a cutoff for differentiating 
low and high levels of control in violent intimate relationships (Johnson, 2008). No studies, however, have reported on the use of a cutoff score for dichotomizing levels of control in IPV.

In addition, there is a need to understand the use of control not only in the context of IPV but also in the cultural system where the violence occurs (Dutton, 1996; Edleson \& Tolman, 1992). Previously, research found that psychological abuse victimization, rather than physical and/or sexual abuse victimization, had a negative impact on Chinese women's mental health (Tiwari et al., 2007). Despite the conjecture that the partners might have used ridiculing and shaming to control the women in the above study, the use of control by the perpetrator was not measured. Nevertheless, the importance of using a culturally sensitive tool to assess IPV was clearly demonstrated.

A number of measures have been used to assess the use of control in intimate relationships, including a 92-item Coercive Control measure (Dutton, Goodman, \& Schmidt, 2005), behavior-specific questions on controlling behaviors by an intimate partner in the WHO multi-country study (Garcia-Moreno et al., 2005), and the Revised Controlling Behaviors Scale (CBS-R; Graham-Kevan \& Archer, 2005). However, none of the measures have been validated for Chinese women and their appropriateness for these women is therefore not known. 
The present study aimed to evaluate the utility of the Chinese version of the CBS-R (hereafter known as the C-CBS-R) as a measure of controlling behaviors in Chinese intimate relationships and to find a cutoff point on the C-CBS-R using a mixed-methods approach. The CBS-R was chosen because of the commonalities identified (private communication, N. Graham-Kevan, 2010) between the domains of the CBS-R and the control tactics reported by abused Chinese women (Tiwari \& Yuen, 2010)

\section{Method}

\section{Design}

The findings presented in this paper are part of a large cohort study of Chinese women's experiences of IPV victimization (Tiwari et al., 2012). The present study adopted a mixed-methods design to elicit qualitatively the women's personal experiences of control in intimate relationships through individual, in-depth, semi-structured, face-to-face interviews. A quantitative approach was also used to evaluate the C-CBS-R through a researcher-administered questionnaire. The study was approved by the Institutional Review Board of the principal investigator's institution.

\section{Participants and Setting}

To be eligible for the study, the participants had to be Chinese women, 18 years of age 
or older, able to communicate in Cantonese or Putonghua (the two main dialects spoken in Hong Kong), and screened positive by the researcher for intimate partner physical violence victimization in the preceding 12 months based on the Chinese Abuse Assessment Screen. A total of 200 women met the inclusion criteria and were recruited for the present study. Recruitment sites were deliberately selected to maximize diversity in the women's IPV victimization experiences. Thus, recruitment sites covered all districts in Hong Kong and included shelters for abused women, community centers operated by non-governmental organizations, and the Family and Child Protective Services Units (FCPSUs) under the Social Welfare Department of the Government of Hong Kong Special Administrative Region. Of the 200 participants, 100 were recruited from the four shelters for abused women and the remaining 100 women were recruited from 7 community centers and 11 FCPSUs.

\section{Measurements}

Qualitative Measures The interviewer sought to elicit information from the participants about the context in which the violence occurred and their experiences of control in their intimate relationships. An opening question was posed to the interviewee: "Can you tell me what it has been like living with your partner all these years?" Prompts were used to help elaborate on the accounts reported, such as: "What do you and your partner do when dealing with matters arising between you 
(such as family income, spending, parenting, in-laws, socializing with friends, going out without the partner)?" / "What do you and your partner do when you two disagree about something?" / "Do you feel that you are free to do what you want?" (with reference to the subject under discussion, such as parenting, spending, socializing, etc.) / "Are there times in your relationship when you feel that your partner is the one who decides what you can or cannot do, and how do you feel when that happens?" / "Are you afraid of your partner?"

\section{Quantitative Measures}

\section{Controlling Behavior A 32-item Revised Controlling Behaviors Scale (CBS-R)}

developed by Graham-Kevan and Archer (private communication, N. Graham-Kevan, 2010) was adopted to measure the use of controlling behaviors by the woman (self-reports) and that by the partner (derived from the woman's report on her partner) (Graham-Kevan \& Archer, 2005); this was created by adding the subscales of using children and minimizing to the original five subscales of economic abuse, coercion and threats, intimidation, emotional abuse, and isolation. Each item of the CBS-R was rated on a 5-point Likert scale ranging from 0 (=never $)$ to 4 (= always). The original CBS-R has demonstrated satisfactory alpha values (Graham-Kevan \& Archer, 2009).

For measuring controlling behaviors in violent Chinese intimate relationships, 
the 32-item English version of the CBS-R was forward translated into traditional

Chinese. To ensure that the meaning of each item was retained, a consensus meeting was held by two of the investigators (AT and GL) who are bilingual (in traditional Chinese and English). It was then back translated into English by a professional translator who was blinded to the original English version of the CBS-R. The back-translated English version was compared with the original English version to ensure equivalence in meaning and expression. Modifications were made as appropriate (first draft). It was then independently reviewed by three Chinese researchers/practitioners with expertise in IPV victimization and perpetration in the Chinese community. All three reviewers found the Chinese CBS-R to be relevant, appropriate, and comprehensive. Only minor revisions were made to some of the wordings of the items as suggested by the reviewers, and the C-CBS-R was revised accordingly (second draft). Cultural adaptation testing was conducted with five abused Chinese women who were invited to complete the C-CBS-R and then undergo cognitive de-briefing with the PI in face-to-face interviews. Further modifications and refinements were made based on the women's feedback on clarity, ease of understanding, and relevance (final draft). Finally, the C-CBS-R was pilot tested for feasibility with another 15 abused Chinese women. No further revisions were made to the C-CBS-R after the pilot test. 
In addition, the Chinese Revised Conflict Tactics Scale (C-CTS2; Chan et al., 2008), Chinese post-traumatic stress disorder (PTSD) Checklist Civilian version (C-PCL-C; Wu, Chan, \& Yiu, 2008), and Chinese Beck Depression Inventory version II (C-BDI-II; Chinese Behavioral Science Society, 2000) were administered to assess participants for intimate partner physical violence victimization, PTSD symptoms, and depressive symptoms, respectively.

\section{Procedure}

The study was conducted between September 2010 and August 2012. In a private room provided by the shelter/community center/FCPSU, an individual, semi-structured interview was conducted and recorded digitally with the woman's permission. In order to elicit private and sensitive information from Chinese women survivors of IPV, we have developed an interviewing technique which allows us to build trust with the interviewee and gain access to her experiences, feelings, and thoughts about her intimate relationship (Tiwari et al., 2011). Furthermore, in addition to the verbal accounts, the interviewer also enhanced the richness of the interview data by keeping field notes, including the non-verbal cues displayed by the woman (such as her facial expression, gesture, posture, and tone of voice), distressing thoughts, feelings, and images. The field notes also included clarifications sought, the interviewer's understanding of the woman's experiences and empathy for the 
woman, inferences drawn from the woman's utterances, and her comments about the accuracy of the inferences. In addition, a questionnaire containing the C-CTS2, C-CBS-R, C-PCL-C, and C-BDI-II was also administered.

\section{Qualitative Data Analysis}

Three of the researchers (AT, GL, and KL) independently undertook a structured analytic process in order to gain a deep understanding of the meaning of the data.

During the process, which took several months, the researchers became deeply immersed in the data (women's accounts and field notes) through repeated reading, intuiting, analyzing, and synthesizing (Streubert \& Carpenter, 2011). In addition, the cyclic nature of questioning and verifying that started in the data collection stage continued throughout the data analysis process. This ensured that the researchers questioned their prior conclusions in the context of what they had discovered at different points in the analysis process. Data analysis took the form of identifying key words and phrases and grouping similar ones into categories. The categories were critically examined and interpretations were made. Similar categories were clustered to form themes. Through questioning, verifying, and reflecting, the researchers related the themes to one another and arrived at a rich description of the phenomenon being investigated, that is, the context in which partner violence took place in Chinese intimate relationships. The rigor of the analysis was ensured by the 
three researchers undertaking repeated rounds of critical discussion and debate, in addition to their own analysis, to assess the accuracy and credibility of the emerging themes.

\section{Statistical Analysis}

The C-CBS-R was analyzed as in Johnson (1999), based on the women's reports of their and their partners' use of controlling behaviors. For each individual, the mean C-CBS-R score was calculated as the total item score divided by the number of applicable items. To determine a cutoff score of the C-CBS-R for dichotomizing high and low levels of controlling behaviors, the participants' qualitative findings (that is, the reported partner violence took place in a context of coercive control [yes] or not [no]) and the quantitative findings (that is, the mean C-CBS-R scores) were inputted for analysis. The sample of 200 participants was randomly split into two halves. For the first half, using the qualitative findings as the benchmark, as suggested by Johnson (2008), we conducted a receiver operating characteristic (ROC) curve analysis. Specifically, the Youden index, that is, sensitivity- (1-specificity), was calculated for different cutoff values, and the cutoff value that maximized the Youden index was taken as optimal. The optimal cutoff value was then validated by using the second half of the sample to obtain the sensitivity, specificity, positive predictive value (PPV), and negative predictive value (NPV). Criterion validity of the 
C-CBS-R cutoff score was assessed by using a t-test to examine the known higher depression level in individuals experiencing partner violence accompanied by coercive control (Dutton, Goodman, \& Schmidt, 2005). The Statistical Package for the Social Sciences (SPSS) version 20.0 was used for the analysis and $95 \%$ confidence intervals were reported where appropriate.

\section{Results}

\section{Participants}

Table 1 shows the demographic characteristics and IPV experiences of the 200 women in the study.

Qualitative findings: The context in which partner violence took place in Chinese intimate relationships

Of the 200 women interviewed, 91 gave vivid accounts of how their partners used, in addition to physical violence, a wide range of behaviors to control them, including denying them money and support, and restricting their contacts with others. The partners' wishes had to be obeyed and failure to do so would result in punishment of a physical (e.g., beating) or sexual (e.g., forced sex) nature. The fear of punishment often deterred the women from doing anything of which (they thought) their partner would not approve. Not only did the women have to account for every minute of their movements, the partners also checked on them frequently (e.g., via cell phone). 
Subjected to frequent and repeated episodes of humiliations, threats, and verbal abuse, the women were living in a state of fear and uncertainty. The lack of financial means, fear of reprisal from the partner, and the actual or threatened embarrassment by the partner in front of their family or friends have also deterred many of the women from visiting their families or socializing with friends. The accounts provided by these 91 women were consistent with Dutton and Goodman's (2005) descriptions of partners' use of coercive control in violent intimate relationships. Such descriptions were not apparent in the accounts provided by the remaining 109 women, whose experiences of IPV were often the result of disagreements over parenting practices, financial difficulties, the partner's alcohol abuse, or his extra-marital affairs.

Quantitative findings: Evaluation of the $C-C B S-R$ and the cutoff score The mean $C$-CBS-R score: Table 2 shows the overall mean C-CBS-R scores and mean scores for each of the five subscales indicating controlling behaviors used by the women and their partners. The alpha values for the C-CBS-R were 799 (women) and .928 (partners).

The cutoff score of the C-CBS-R: The results of the ROC curve analysis for the first half of the data are shown in Figure 1. The area under the curve (AUC) is .991 (95\% CI: .982 to .999; $\mathrm{p}<.001)$. The sensitivity, specificity, PPV, NPV, and Youden's 
index of the different cutoff mean scores on the C-CBS-R to identify the use of coercive control are displayed in Table 3. The cutoff score of 1.145, which has the highest Youden's index, was selected. The chosen cutoff score was then applied to the second half of the data to evaluate the accuracy of the cutoff score. With a sensitivity of $96 \%$ (95\% CI $=88.1 \%$ to $100 \%)$, specificity of $95 \%(95 \% \mathrm{CI}=88.9 \%$ to $97.1 \%)$, and PPV and NPV of $87 \%(95 \% \mathrm{CI}=65.0 \%$ to $89.0 \%)$ and $99 \%(95 \% \mathrm{CI}$ $=97.3 \%$ to $100 \%$ ), respectively, the score of 1.145 was found to be an optimal cutoff. The correct classification rate was $93 \%(95 \% \mathrm{CI}=89.3 \%$ to $96.7 \%)$. Criterion validity of the C-CBS-R cutoff score: By applying the C-CBS-R cutoff score of 1.145 to the women's reports of mental health effects of IPV, it was found that women experiencing partner violence accompanied by the partner's use of a high level of controlling behaviors (i.e., > 1.145) reported significantly higher depressive symptoms (31.72 vs. $11.61 ; p<.001)$ and PTSD symptoms $(60.24$ vs. $33.89 ; p<.001)$ compared to those women whose experience of partner violence was not accompanied by the partner's use of a high level of controlling behaviors.

\section{Discussion}

The present study was the first to report on the utility of the C-CBS-R as a measure of controlling behaviors, and a cutoff point for distinguishing high from low levels of controlling behaviors in Chinese intimate relationships. Using an agency sample 
(abused women recruited from abused women shelters) and a community sample (abused women recruited from community centers and FCPSUs) in Hong Kong, we conducted a mixed-methods study, as suggested by Johnson (2008), to evaluate the utility of the C-CBS-R and to validate the cutoff score of the C-CBS-R both qualitatively and quantitatively. We found that the C-CBS-R had utility as a measure of controlling behaviors in violent Chinese intimate relationships. Additionally, we successfully demonstrated that a cutoff score of 1.145 on the C-CBS-R was an optimal point for distinguishing high from low levels of controlling behaviors. Evidence of criterion validity was also found, with the C-CBS-R scores positively correlated with scores of PTSD and depressive symptoms.

An advantage of a cutoff score on the C-CBS-R is that frontline service providers can work out whether the reported controlling behaviors are indicative of a high or low level of control in the context of violent intimate relationships. This would allow them to make early identification of those most at risk of further violence and/or injury so that timely and potentially effective interventions may be initiated. For example, if partner violence is found to be accompanied by a high level of controlling behaviors by the partner, ending the relationship does not necessarily end the violence. Indeed, the threat to the perpetrator's control as a result of the separation may even lead to an escalation of the use of control (Bachman \& Saltzman, 
1995). In such cases, ensuring the safety of the woman and other members of her family must be a priority in addition to helping the woman to leave the relationship. In the present study, a partner's use of a high level of controlling behaviors in a violent Chinese intimate relationship was found to be correlated with more severe depressive and PTSD symptoms. Our finding is consistent with Johnson and Leone's (2005) analysis of 4967 married women in the National Violence Against Women Survey, which revealed that IPV survivors experiencing a high level of coercive control had significantly higher scores of depressive and PTSD symptoms. In a well-documented meta-analysis by Golding (1999), the adverse impact of IPV on survivors' mental health was clearly demonstrated, with depression and PTSD being the two most common mental health sequelae of IPV. Our present study has confirmed and further extended the findings of Golding's review by delineating the role played by controlling behaviors in the link between IPV and mental health outcomes. Future research could further investigate the mediating effect of controlling behaviors on the association between IPV and survivors' mental health.

The qualitative data elicited from the 200 interviews are a strength of this study and have provided rich information about the context of violent Chinese intimate relationships hitherto unreported. The women have confirmed, as previously suggested for non-Chinese IPV survivors (e.g., Dutton, 1996; Edleson \& Tolman, 
1992), that the measurement of violent acts alone cannot adequately explain the violence in intimate relationships. Indeed, for nearly half of the women in this study, their partners' violent behaviors were not mindless acts but were a means to gain control over them, as previously suggested by Johnson (2008).

Limitations of this study include the use of self-reports, which are liable to erroneous recall and social desirability, thus affecting the accuracy of the women's reports. Despite recruiting participants from all districts in Hong Kong, the small sample size and convenience sampling limit the generalizability of the findings. The use of a prospective design with a larger representative sample may improve the accuracy of the data collected.

In conclusion, using a qualitative-quantitative design in a sample of abused Chinese women, we have shown that the C-CBS-R has utility as a measure of controlling behaviors, with a cutoff score to distinguish high from low levels of control in violent Chinese intimate relationships.

\section{References}

Bachman, R., Saltzman, L.E. (1995). Violence against women: Estimates from the Redesigned Survey, special report. Washington, D.C.:U.S. Department of Justice.

Cattell, R. B. (1978). The scientific use of factor analysis in behavioral and life sciences. New York: Plenum Press. 
Chan, K.L., Brownridge, D.A., Tiwari, A., Fong, D.Y.T., Leung, W.C. (2008)

Understanding violence against Chinese women in Hong Kong: An analysis of risk factors with a special emphasis on the role of in-law conflict. Violence Against Women, 14(11): 1295-1312.

Chinese Behavioral Science Society (2000). The Chinese version of the Beck Depression Inventory ( $2^{\text {nd }}$ ed.). Licensed Chinese Translation. The Psychological Corporation. New York: Harcourt Brace.

Dutton, M. A. (1996). Battered women's strategic response to violence: The role of context. In J. Edleson \& Z. Eiskovitz (Eds.), Future interventions with battered women and their families (pp. 105-124). Thousand Oaks, CA: Sage.

Dutton, M. A., \& Goodman, L. A. (2005). Coercion in intimate partner violence: Toward a new conceptualization. Sex Roles, 52(11/12), 743-756.

Dutton, M. A., Goodman, L., \& Schmidt, R. J. (2005). Development and validation of a coercive control measure for intimate partner violence. Final technical report to National Institute of Justice. COSMOS Corporation.

Edleson, J. L., \& Tolman, R. M. (1992). Intervention for men who batter: An ecological approach. Newbury Park: Sage.

Garcia-Moreno, C., Jansen, H., Watts, C., Ellsberg, M., Heise, L. (2005). WHO multi-country study on women's health and domestic violence against women: 
Initial results on prevalence, health outcomes and women's responses. Geneva:

WHO.

Golding, J.M. (1999). Intimate Partner Violence as a Risk Factor for Mental Disorders:

A Meta-Analysis. Journal of Family Violence, 14(2), 99-132.

Graham-Kevan, N., \& Archer, J. (2003). Physical aggression and control in heterosexual relationships: the effect of sampling. Violence and Victims, 18(2), $181-196$

Graham-Kevan, N., \& Archer, J. (2005). Investigating three explanations of women's relationship aggression. Psychology of Women Quarterly, 29, 270-277.

Graham-Kevan, N., \& Archer, J. (2009). Control tactics and partner violence in heterosexual relationships. Evolution and Human Behavior, 30, 445-452.

Johnson, M. P. (2008). A typology of domestic violence: Intimate terrorism, violent resistance, and situational couple violence. Boston: Northeastern University Press.

Johnson, M. P. \& Leone, J. M. (2005). The differential effects of intimate terrorism and common couple violence: Findings from the National Violence Against women Survey. Journal of Family Issues, 26(3), 322-349.

Punj, G. \& Stewart, D. W. (1983). Cluster analysis in marketing research: Review and suggestions for application. Journal of marketing research: JMR, 20, 134 - 148. 
Streubert, H. J. \& Carpenter, D. R. (2011). Qualitative research in nursing: Advancing the humanistic imperative. Philadelphia: Wolters Kluwer Health/Lippincott Williams \& Wilkins.

Tiwari, A., Fong, D. Y. T., Chan, K. L., Leung, W. C., Parker, B., \& Ho, P. C. (2007). Identifying intimate partner violence: Comparing the Chinese abuse assessment screen with the Chinese revised conflict tactics scales. British Journal of Obstetrics and Gynaecology, 114(9), 1065-1071.

Tiwari, A., Fong, D. Y. T. \& Lam, L. L. (2011). Interviewing Chinese women survivors of domestic violence and sexual assault for research purpose. Paper presented at Ending Domestic \& Sexual Violence Conference, Portsmouth, NH.

Tiwari, A., Lam, L. L. \& Hong, W. L. (2012). Differentiating Intimate Terrorism and Situational Couple Violence: Process and Outcomes. Paper presented at the $18^{\text {th }}$ Annual Conference of NNVAWI, Charlottesville, VA.

Tiwari, A., Yuen, K.H. (2010). Psychological abuse in Chinese women: A qualitative study. Family \& Intimate Partner Violence Quarterly, 2, 293-302.

Wu, K. K., Chan, S. K., \& Yiu, V. F. (2008). Psychometric properties and confirmatory factor analysis of the posttraumatic stress disorder checklist for Chinese survivors of road traffic accidents. Hong Kong Journal of Psychiatry, $18(4), 144-155$. 


\section{Tables and Figures}

Table 1. Demographic characteristics and IPV victimization

\begin{tabular}{|c|c|}
\hline Demographic characteristics & $\mathbf{N}(\%)$ \\
\hline \multicolumn{2}{|l|}{ Place of recruitment } \\
\hline Shelters & $100(50 \%)$ \\
\hline Community settings & $100(50 \%)$ \\
\hline \multicolumn{2}{|l|}{ Age (mean [SD]) } \\
\hline Women & $41.63 \pm 11.42$ \\
\hline Partners & $49.61 \pm 12.80$ \\
\hline \multicolumn{2}{|l|}{ Employment status } \\
\hline \multicolumn{2}{|l|}{ Women } \\
\hline Employed & $58(29.0 \%)$ \\
\hline Housewife & $132(66.0 \%)$ \\
\hline \multicolumn{2}{|l|}{ Partners } \\
\hline Employed & $124(62.9 \%)$ \\
\hline Unemployed & $43(21.8 \%)$ \\
\hline Retired & $30(15.2 \%)$ \\
\hline \multicolumn{2}{|l|}{ Marital status } \\
\hline Single & $5(2.5 \%)$ \\
\hline Married/cohabiting & $95(47.5 \%)$ \\
\hline Separated/divorced & $100(50.0 \%)$ \\
\hline \multicolumn{2}{|l|}{ Women's place of birth } \\
\hline Hong Kong & $46(23.0 \%)$ \\
\hline Mainland China & $154(77.0 \%)$ \\
\hline \multicolumn{2}{|l|}{ Number of children } \\
\hline 0 & $10(5.0 \%)$ \\
\hline 1 & $76(38.0 \%)$ \\
\hline 2 & $80(40.0 \%)$ \\
\hline 3 or more & $34(17.0 \%)$ \\
\hline Financial hardship (past year) & $132(66.0 \%)$ \\
\hline \multicolumn{2}{|l|}{ IPV victimization (past year) } \\
\hline Psychological abuse & $200(100 \%)$ \\
\hline Physical violence & $200(100 \%)$ \\
\hline Sexual coercion & $66(33.2 \%)$ \\
\hline Physical injury & $163(81.5 \%)$ \\
\hline Medical services sought & $103(51.5 \%)$ \\
\hline
\end{tabular}


Table 2. Mean C-CBS-R scores and standard deviations (S.D.) indicating women's and partners' use of controlling behaviors

\begin{tabular}{|c|c|c|c|c|c|}
\hline & \multicolumn{2}{|c|}{ Partners } & \multicolumn{2}{|c|}{ Women } & \multirow{2}{*}{$\mathrm{t}$} \\
\hline & Mean & S.D. & Mean & S.D. & \\
\hline Economic abuse & 1.549 & 1.377 & 0.060 & 0.255 & $1.489 * * *$ \\
\hline Coercion and threats & 0.929 & 1.019 & 0.251 & 0.390 & $0.679 * * *$ \\
\hline Intimidations & 1.427 & 1.029 & 0.253 & 0.365 & $1.174 * * *$ \\
\hline Emotional abuse & 1.395 & 1.172 & 0.130 & 0.367 & $1.265 * * *$ \\
\hline Isolation & 1.214 & 1.496 & 0.205 & 0.586 & $1.010 * * *$ \\
\hline Using Children & 1.194 & 0.983 & 0.403 & 0.502 & $0.791 * * *$ \\
\hline Minimizing & 0.732 & 1.083 & 0.005 & 0.067 & $0.727 * * *$ \\
\hline Total & 1.233 & 0.935 & 0.240 & 0.215 & $0.944 * * *$ \\
\hline
\end{tabular}

$*$ p-values $<0.05$

Figure 1. Receiver operating characteristic (ROC) curve for using the C-CBS-R to assess coercive control

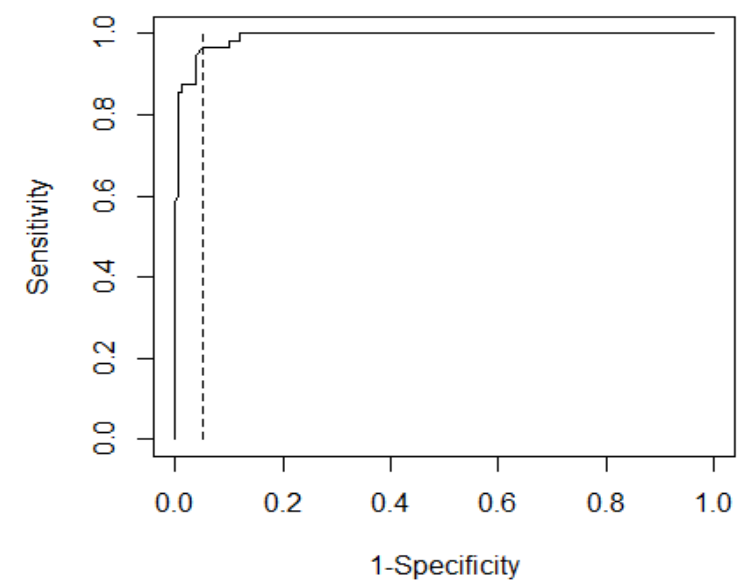

Table 3. The sensitivity, specificity, positive predictive value (PPV), negative predictive value (NPV), and Youden's index of the C-CBS-R at different cutoff mean scores

\begin{tabular}{cccccc}
\hline Cutoff Score & Sensitivity & Specificity & PPV & NPV & Youden's index \\
\hline 1.087 & 0.964 & 0.925 & 0.815 & 0.987 & 0.889 \\
1.113 & 0.964 & 0.944 & 0.855 & 0.987 & 0.908 \\
$\mathbf{1 . 1 4 5}$ & $\mathbf{0 . 9 6 4}$ & $\mathbf{0 . 9 5 0}$ & $\mathbf{0 . 8 6 9}$ & $\mathbf{0 . 9 8 7}$ & $\mathbf{0 . 9 1 4}$ \\
1.258 & 0.945 & 0.963 & 0.897 & 0.981 & 0.908 \\
1.387 & 0.927 & 0.963 & 0.895 & 0.975 & 0.890 \\
\hline
\end{tabular}


